\title{
Weighted Measurement Fusion Quantized Filtering with Bandwidth Constraints and Missing Measurements in Sensor Networks
}

\author{
Jian Ding, Jing Ma, and Shuli Sun \\ Department of Automation, Heilongjiang University, Harbin 150080, China \\ Correspondence should be addressed to Shuli Sun; sunsl@hlju.edu.cn
}

Received 14 September 2013; Accepted 29 December 2013; Published 4 February 2014

Academic Editor: Wendong Xiao

Copyright (C) 2014 Jian Ding et al. This is an open access article distributed under the Creative Commons Attribution License, which permits unrestricted use, distribution, and reproduction in any medium, provided the original work is properly cited.

\begin{abstract}
This paper is concerned with the estimation problem of a dynamic stochastic variable in a sensor network, where the quantization of scalar measurement, the optimization of the bandwidth scheduling, and the characteristic of transmission channels are considered. For the imperfect channels with missing measurements in sensor networks, two weighted measurement fusion (WMF) quantized Kalman filters based on the quantized measurements arriving at the fusion center are presented. One is dependent on the known message of whether a measurement is received. The other is dependent on the probability of missing measurements. They have the reduced computational cost and same accuracy as the corresponding centralized fusion filter. The approximate solution for the optimal bandwidth-scheduling problem is given under a limited bandwidth constraint. Furthermore, the vector measurement case is also discussed. The simulation research shows the effectiveness.
\end{abstract}

\section{Introduction}

In recent years, sensor networks have been widely investigated in decentralized estimation, detection, and control due to the significant applications in environmental monitoring, intelligent transportation, space exploration, and so forth [1]. In wireless sensor networks (WSN), a large number of sensors are spatially distributed to monitor the signal of interest. Each sensor makes a measurement of the signal and transmits it to the fusion center (data processing center). Due to a bandwidth constraint, each sensor is only able to transmit a finite number of bits. So the measurement must be quantized to adapt the limited bandwidth before it is transmitted. Due to the imperfection of networks, the quantized measurement can be lost during the transmission. Then the fusion centre will use the quantized measurements received to obtain a fusion estimate of the signal. WSN introduce many interesting research topics such as information fusion [2], network lifetime maximization [3], sensor coverage or scheduling [4], and optimization with bandwidth or energyefficient constraints [5].

Various algorithms have been proposed for network estimation, detection, and control [5-18]. Decentralized detection is investigated in a sensor network where the communication channels between sensors and the fusion centre are bandwidth constrained [5]. Several distributed estimators for parameters have been designed in the presence of additive sensor noise [6-10]. A universal decentralized estimator taking into account local SNR and channel path loss in sensor networks is studied [11] where the power scheduling optimization is solved based on the Karush-Kuhn-Tucker (KKT) condition. Quantization approach in many references above is to quantize the sensor's measurements directly. A distributed estimation approach based on the sign of innovations (SOI) is developed in [12] where only the transmission of a single bit per measurement is required. However, the cost of saving more communication is more accuracy loss. As a generalization of [12], a multilevel quantized innovation filter is presented $[13,14]$. The estimation and control based on the logarithm quantization approach are studied in [15, 16]. Quantized Kalman filters based on quantized scalar measurements and innovations are presented for perfect channels in sensor networks [17], respectively. However, the quantized estimation for imperfect channels with missing measurements is not taken into consideration. A centralized 
fusion quantized filter dependent on the packet dropout rate is designed for sensor networks with packet dropouts [18]. However, it has the expensive computational burden due to the augmented measurements.

In this paper, the quantized estimation problem for a dynamic stochastic variable is studied in a sensor network. Due to the limited bandwidth constraint, the measurement of sensors is quantized uniformly according to a given optimal bandwidth scheduling. During the transmission of quantized measurements, there are possible losses due to imperfect channels. Due to the large number of data, the fusion center compresses the received measurements to produce a reduced dimensional fused measurement, based on which, two weighted measurement fusion quantized filters are presented. One is dependent on the knowledge of whether a packet is received. The other is dependent on the probabilities of missing measurements. The front has the better accuracy since more messages are used. They have the same accuracy as the corresponding centralized fusion filters.

\section{Problem Formulation}

Consider the discrete-time system in a sensor network with $N$ sensors

$$
\begin{gathered}
x(t+1)=\Phi(t) x(t)+\Gamma(t) w(t) \\
y_{i}(t)=h_{i}(t) x(t)+v_{i}(t), \quad i=1,2, \ldots, N,
\end{gathered}
$$

where $x(t) \in R^{n}$ is the state to be estimated, $y_{i}(t) \in R$ is the scalar measurement of the $i$ th sensor, $N$ is the number of sensors, and $\Phi(t), \Gamma(t), h_{i}(t)$ are time-varying matrices with appropriate dimensions.

Assumption 1. $w(t) \in R^{r}$ and $v_{i}(t) \in R, i=1,2, \ldots, N$ are uncorrelated white noises with zero mean and variances $Q_{w}(t)$ and $\sigma_{v_{i}}^{2}(t)$. The initial value $x(0)$ with mean $\mu_{0}$ and variance $P_{0}$ is uncorrelated with $w(t)$ and $v_{i}(t)$.

Assumption 2. $y_{i}(t) \in\left[\underline{U}_{i}, \bar{U}_{i}\right]$, where $\underline{U}_{i}$ and $\bar{U}_{i}, i=$ $1,2, \ldots, N$, are known constants.

The estimation problem considered is shown in Figure 1. Each sensor makes a measurement $y_{i}(t)$. Due to the limited bandwidth, it is quantized to produce a quantized measurement $m_{i}(t)=q\left(y_{i}(t)\right)$ where $q(\cdot)$ is a quantized function. Then, $m_{i}(t)$ is transmitted to the fusion center by an imperfect channel where there are possible packet losses. We introduce a Bernoulli distributed random variable $\gamma_{i}(t)$ with the probabilities $\operatorname{Prob}\left\{\gamma_{i}(t)=1\right\}=\alpha_{i}$ and $\operatorname{Prob}\left\{\gamma_{i}(t)=0\right\}=$ $1-\alpha_{i}$ to describe the phenomena of missing measurements. Namely, the data received by the fusion center is $m_{i}^{\prime}(t)=$ $\gamma_{i}(t) m_{i}(t)$, where $\gamma_{i}(t)=1$ means the quantized measurement is received and $\gamma_{i}(t)=0$ means loss. At last, the fusion center will combine the received data $m_{i}^{\prime}(t)$ to give a final estimate for state $x(t)$. We assume that the fusion center knows all the parameters of system (1). If there is a sufficient bandwidth to be supplied and the channel is perfect, that is, in the case of $m_{i}^{\prime}(t)=y_{i}(t)$, the standard Kalman filter can be used [19]. If the bandwidth is limited and the channel is perfect, that is, in the case of $m_{i}^{\prime}(t)=m_{i}(t)$, the fusion center will make the estimate based on the received measurements $\left\{m_{i}(t), i=\right.$ $1,2, \ldots, N\}$. Otherwise, the fusion center has to make the estimate based on the received measurements $\left\{m_{i}^{\prime}(t), i=\right.$ $1,2, \ldots, N\}$.

Our aim in this paper is to find the weighted measurement fusion quantized Kalman filters (WMF-QKF) under the limited bandwidth by imperfect channels. Two kinds of filters are designed. One is dependent on the values of $\gamma_{i}(t)$, the other is dependent on the probability of $\gamma_{i}(t)$.

\section{WMF-QKF}

3.1. Quantization and Bandwidth Scheduling. We adopt the uniform quantization strategy in [11]. Measurement $y_{i}(t)$ is quantized to $m_{i}(t)$ with the length of $b_{i}(t)$ bits, where $b_{i}(t)$ is to be determined later. We have $2^{b_{i}(t)}$ quantization points spaced uniformly within the interval $\left[\underline{U}_{i}, \bar{U}_{i}\right]$. The quantization noise $n_{i}(t)=m_{i}(t)-y_{i}(t)$ is uncorrelated white noise with zero mean and variance $\sigma_{n_{i}}^{2}(t)=\mathrm{E}\left(n_{i}(t)\right)^{2}=$ $\mathrm{E}\left(m_{i}(t)-y_{i}(t)\right)^{2} \leq \delta_{i}^{2}(t)$ where $\delta_{i}^{2}(t)=\left(\bar{U}_{i}-\underline{U}_{i}\right)^{2} /\left[4\left(2^{b_{i}(t)}-\right.\right.$ $1)^{2}$ ]. Furthermore, $n_{i}(t), v_{i}(t), i=1,2, \ldots, N$, and $w(t)$ are uncorrelated with each other.

In sensor networks, the whole bandwidth of communication channels is bounded. Let $B$ be the bits of the whole bandwidth and let $b_{i}(t)$ be the bits scheduled to the $i$ th sensor. To obtain the good estimation performance under the constraint of bounded bandwidths, we adopt the following bandwidth scheduling strategy [17]:

$$
\begin{array}{ll}
\min & \sum_{i=1}^{N} \frac{h_{i}(t) h_{i}^{T}(t)}{\sigma_{v_{i}}^{2}(t)} \delta_{i}^{2}(t) \\
\text { s.t. } & \sum_{i=1}^{N} b_{i}(t) \leq B, \quad b_{i}(t) \geq 0, \quad i=1,2, \ldots, N,
\end{array}
$$

where $h_{i}(t) h_{i}^{T}(t) / \sigma_{v_{i}}^{2}(t)$ is the SNR (signal to noise ratio). Then the optimal solution of $b_{i}(t)$ is given as

$$
\begin{gathered}
b_{i}(t)=\left[\log _{2}\left(\frac{\sqrt{\ln 2 h_{i}(t) h_{i}^{T}(t)}\left(\bar{U}_{i}-\underline{U}_{i}\right)}{\sigma_{v_{i}}(t) \sqrt{2 \lambda(t)}}\right)\right], \\
\lambda(t)=\frac{\ln 2 / 2 \prod_{i=1}^{N}\left(h_{i}(t) h_{i}^{T}(t)\left(\bar{U}_{i}-\underline{U}_{i}\right)^{2} / \sigma_{v_{i}}^{2}(t)\right)^{1 / N}}{2^{2 B / N}},
\end{gathered}
$$

where the symbol $[\cdot]$ denotes the least integer greater than $\cdot$

\subsection{Design of Two Kinds of WMF-QKF}

3.2.1. Filter Design Dependent on Values of $\gamma_{i}(t)$. When the values of $\gamma_{i}(t)$ are known, that is, we know whether a packet is received or lost, which can be carried out by the information of time stamps, letting $L(t)$ be the number of measurements received by the fusion center at $t$ time, then we have the augmented measurement equation in the fusion center:

$$
m^{\prime}(t)=h(t) x(t)+\eta(t),
$$




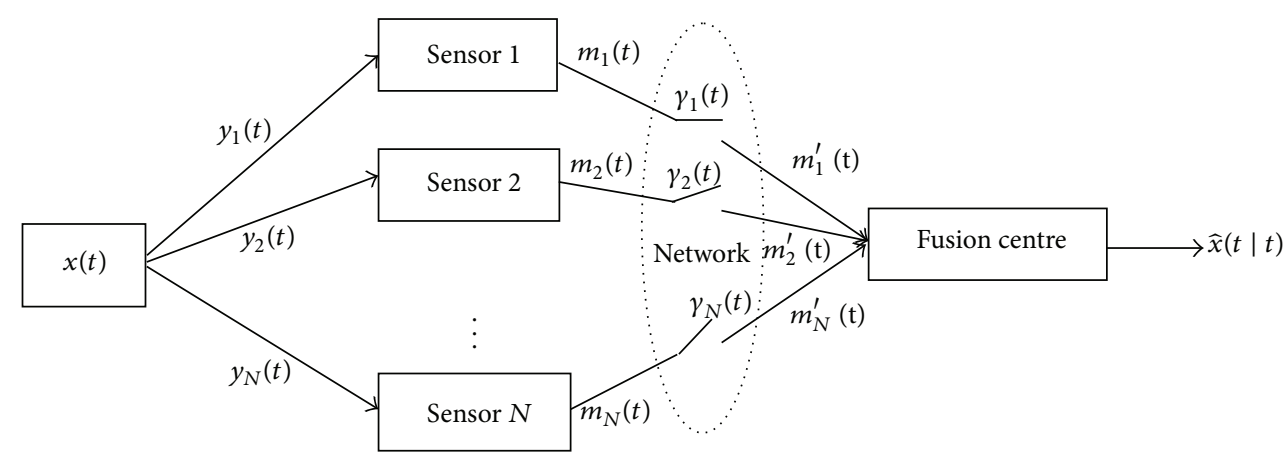

FIGURE 1: Distributed state estimation scheme based on quantized observations.

where the augmented quantized measurement received by the fusion center is $m^{\prime}(t)=\left[m_{k_{1}(t)}^{\prime}(t), m_{k_{2}(t)}^{\prime}(t), \ldots\right.$, $\left.m_{k_{L(t)}(t)}^{\prime}(t)\right]^{T}, 1 \leq k_{1}(t)<\cdots<k_{L(t)}(t) \leq N$ and the integer $k_{i}(t)$ denotes the $k_{i}(t)$ th sensor that arrives at the fusion center. The augmented measurement matrix is $h(t)=$ $\left[h_{k_{1}(t)}^{T}(t), h_{k_{2}(t)}^{T}(t), \ldots, h_{k_{L(t)}(t)}^{T}(t)\right]^{T}$ and the noise is $\eta(t)=$ $\left[\eta_{k_{1}(t)}(t), \eta_{k_{2}(t)}(t), \ldots, \eta_{k_{L(t)}(t)}(t)\right]^{T}$ with zero mean and variance matrix $Q_{\eta}(t)=\operatorname{diag}\left(\sigma_{\eta_{k_{1}(t)}}^{2}(t), \sigma_{\eta_{k_{2}(t)}}^{2}(t), \ldots, \sigma_{\eta_{k_{L}(t)}(t)}^{2}(t)\right)$ where $\eta_{k_{i}(t)}(t)=v_{k_{i}(t)}(t)+n_{k_{i}(t)}(t)$ are uncorrelated with one another, with zero mean and variance $\sigma_{\eta_{k_{i}(t)}}^{2}(t)=\sigma_{v_{k_{i}(t)}}^{2}(t)+$ $\sigma_{n_{k_{i}(t)}}^{2}(t) \leq \sigma_{v_{k_{i}(t)}}^{2}(t)+\delta_{k_{i}(t)}^{2}(t)$. We approximately consider the measurement noise $\eta(t)$ to be the white noise. Then, the Kalman filtering can be used for the augmented systems (1) and (5) where the upper bound of variance of the quantized noise is used. However, the expensive computational cost is required due to the high-dimensional augmented measurement when the data of a large number of sensors arrive at the fusion center. To reduce the computational cost, we will present the WMF filter in the following text.

When $L(t) \geq 1$, that is, there are measurement data arriving at the fusion center at time $t$, then we can obtain the filter according to the following three cases.

(a) If $h(t)$ is full row rank, we can apply the standard Kalman filtering algorithm to obtain the fusion filter.

(b) If $h(t)$ is full column rank, we have that $h^{T}(t) Q_{\eta}^{-1}(t) h(t)$ is nonsingular. Then the WMF measurement equation is given as follows:

$$
\bar{m}(t)=x(t)+\bar{\eta}(t),
$$

where $\bar{m}(t)=\left[h^{T}(t) Q_{\eta}^{-1}(t) h(t)\right]^{-1} h^{T}(t) Q_{\eta}^{-1}(t) m^{\prime}(t), \bar{\eta}(t)=$ $\left[h^{T}(t) Q_{\eta}^{-1}(t) h(t)\right]^{-1} h^{T}(t) Q_{\eta}^{-1}(t) \eta(t), \bar{Q}_{\eta}^{-1}(t)=\left[h^{T}(t) Q_{\eta}^{-1}(t)\right.$ $h(t)]^{-1}$.

Then based on systems (1) and (6), we can apply the standard Kalman filtering algorithm to obtain the fusion filter.

(c) If $h(t)$ is not full rank, that is, $h^{T}(t) Q_{\eta}^{-1}(t) h(t)$ is singular, letting $\operatorname{rank}\{h(t)\}=p(t), p(t) \leq \min \{n, L(t)\}$, then there is full-rank decomposition [20]; that is,

$$
h(t)=f(t) \bar{h}(t),
$$

where $f(t) \in R^{L(t) \times p(t)}$ is full column rank and $\bar{h}(t) \in R^{p(t) \times n}$ is full row rank. $f^{T}(t) Q_{\eta}^{-1}(t) f(t)$ is a nonsingular matrix. So, we have the WMF measurement equation as

$$
\overline{\bar{m}}(t)=\bar{h}(t) x(t)+\overline{\bar{\eta}}(t)
$$

where $\overline{\bar{m}}(t)=\left[f^{T}(t) Q_{\eta}^{-1}(t) f(t)\right]^{-1} f^{T}(t) Q_{\eta}^{-1}(t) m^{\prime}(t), \overline{\bar{\eta}}(t)=$ $\left[f^{T}(t) Q_{\eta}^{-1}(t) f(t)\right]^{-1} f^{T}(t) Q_{\eta}^{-1}(t) \eta(t), Q_{\bar{\eta}}(t)=\left[f^{T}(t) Q_{\eta}^{-1}(t)\right.$ $f(t)]^{-1}$.

Then based on systems (1) and (8), we can apply the standard Kalman filtering algorithm to obtain the fusion filter.

When $L(t)=0$, that is, there are no measurement data arriving at the fusion center at time $t$, then, the Kalman predictor is used based on the last estimator.

Remark 3. From (6) and (8), we can know that the dimension of the compressed measurement $\bar{m}(t)$ or $\overline{\bar{m}}(t)$ is not greater than $\min \{n, L(t)\}$. When the number of sensors arriving at the fusion center is large, that is, $L(t) \gg n$, the proposed WMFQKF with the computational order of magnitude $O\left(p^{3}(t)\right)$ can obviously reduce the computational cost compared to the centralized fusion filter with the computational order of magnitude $O\left(L^{3}(t)\right)$. However, they have the same accuracy; that is, WMF-QKF has the global optimality [20].

3.2.2. Filter Design Dependent on Probabilities of $\gamma_{i}(t)$. In this section, we will design the filter dependent on the probabilities of $\gamma_{i}(t)$. At each time, the measurement of each sensor arriving at the fusion center can be expressed as follows:

$$
\begin{aligned}
m_{i}^{\prime}(t)= & \gamma_{i}(t) m_{i}(t) \\
= & \gamma_{i}(t) h_{i}(t) x(t)+\gamma_{i}(t) v_{i}(t) \\
& +\gamma_{i}(t) n_{i}(t), \quad i=1,2, \ldots, N
\end{aligned}
$$

which can be rewritten as

$$
m_{i}^{\prime}(t)=\alpha_{i} h_{i}(t) x(t)+\zeta_{i}(t), \quad i=1,2, \ldots, N,
$$

where $\zeta_{i}(t)=\left(\gamma_{i}(t)-\alpha_{i}\right) h_{i}(t) x(t)+\gamma_{i}(t) v_{i}(t)+\gamma_{i}(t) n_{i}(t), i=$ $1,2, \ldots, N$ are uncorrelated white noises with zero mean and 
variances $\sigma_{\zeta_{i}}^{2}(t)=\alpha_{i}\left(1-\alpha_{i}\right) h_{i}(t) q(t) h_{i}^{T}(t)+\alpha_{i} \sigma_{v_{i}}^{2}(t)+\alpha_{i} \sigma_{n_{i}}^{2}(t)$; $q(t)=\mathrm{E}\left[x(t) x^{T}(t)\right]$ is the state second-order moment which can be computed by $q(t+1)=\Phi(t) q(t) \Phi^{T}(t)+\Gamma(t) Q_{w}(t) \Gamma^{T}(t)$ from (1).

Then the augmented measurements can be expressed as

$$
M^{\prime}(t)=\widetilde{h}(t) x(t)+\zeta(t)
$$

where $M^{\prime}(t)=\left[\begin{array}{llll}m_{1}^{\prime}(t) & m_{2}^{\prime}(t) & \cdots & m_{N}^{\prime}(t)\end{array}\right]^{T}$, $\tilde{h}(t)=\left[\begin{array}{llll}\alpha_{1} h_{1}(t) & \alpha_{2} h_{2}(t) & \cdots & \alpha_{N} h_{N}(t)\end{array}\right]^{T}, \zeta(t)=$ $\left[\begin{array}{llllll}\zeta_{1}(t) & \zeta_{2}(t) & \cdots & \zeta_{N}(t)\end{array}\right]^{T} ; \quad \zeta(t) \quad$ and $\quad w(t)$ are uncorrelated. The variance matrix of $\zeta(t)$ is $Q_{\zeta}(t)=$ $\operatorname{diag}\left(\sigma_{\zeta_{1}}^{2}(t) \quad \sigma_{\zeta_{2}}^{2}(t) \cdots \sigma_{\zeta_{N}}^{2}(t)\right)$ where the symbol $\operatorname{diag}(\cdot)$ denotes the diagonal matrix.

According to the different cases that the matrix $\widetilde{h}(t)$ is full row-rank, full column-rank, or not full-rank, we can obtain the WMF-QKF dependent on probabilities of $\gamma_{i}(t)$ similar to design of the above subsection.

Remark 4. Two kinds of WMF-QKFs have been proposed. The filter dependent on the values of $\gamma_{i}(t)$ (WMF-QKFV) has better accuracy than that dependent on the probabilities of $\gamma_{i}(t)$ (WMF-QKFP) since more information is used. However, WMF-QKFV requires the online computation since it is dependent on the stochastic variable $\gamma_{i}(t)$ at each time. WMFQKFP can be computed offline since it is only dependent on the probabilities. Moreover, WMF-QKFP has the reduced online computational cost than WMF-QKFV.

3.3. Multiple Dimension Measurement Case. WMF-QKF with optimization problems has been solved for systems with scalar measurement in Sections 3.1 and 3.2. In this section, we consider the WMF-QKF for systems with multiple dimension measurements. We consider the system

$$
\begin{gathered}
x(t+1)=\Phi(t) x(t)+\Gamma(t) w(t) \\
y_{i}(t)=H_{i}(t) x(t)+v_{i}(t), \quad i=1,2, \ldots, N,
\end{gathered}
$$

where $y_{i}(t) \in R^{q_{i}}$ is the measurement vector of the $i$ th sensor; other variables have the same definitions as Section 2. $H_{i}(t)$ is full row rank. We make the following assumptions.

Assumption 5. $w(t) \in R^{r}$ and $v_{i}(t) \in R^{q_{i}}, i=1,2, \ldots, N$, are uncorrelated white noises with zero mean and variance matrices $Q_{w}(t)$ and $Q_{v_{i}}(t)$, and their each component is uncorrelated with each other; that is, $Q_{w}(t)$ and $Q_{v_{i}}(t)$ are diagonal matrices.

Assumption 6. $y_{i}^{(k)}(t) \in\left[\underline{U}_{i}^{(k)}, \bar{U}_{i}^{(k)}\right], k=1,2, \ldots, q_{i} ; i=$ $1,2, \ldots, N$, where $y_{i}^{(k)}(t)$ is the $k$ th component of observation vector $y_{i}(t)$ and $\underline{U}_{i}^{(k)}$ and $\bar{U}_{i}^{(k)}$ are known constants.

The system structure is similar to Figure 1. For each component $y_{i}^{(k)}(t)$ of measurement $y_{i}(t)$ from the $i$ th sensor, we quantize each component $y_{i}^{(k)}(t)$ to $m_{i}^{(k)}(t)$ with the length of $b_{i}^{(k)}(t)$ bits according to the quantized approach in
Section 3.1. Let the quantized noise be $n_{i}^{(k)}(t)=m_{i}^{(k)}(t)-$ $y_{i}^{(k)}(t)$; then the variance of the quantized noise $n_{i}^{(k)}(t)$ is $\sigma_{n_{i}^{(k)}}^{2}(t) \leq \delta_{n_{i}^{(k)}}^{2}(t), \delta_{n_{i}^{(k)}}^{2}(t)=\left(\bar{U}_{i}^{(k)}-\underline{U}_{i}^{(k)}\right)^{2} /\left[4\left(2^{b_{i}^{(k)}(t)}-1\right)^{2}\right]$. Furthermore, $n_{i}^{(k)}(t), v_{j}^{(l)}(t), i, j=1,2, \ldots, N ; k=1,2, \ldots, q_{i}$; $l=1,2, \ldots, q_{j}$, and $w(t)$ are uncorrelated with each other. Then, similar to scalar measurement case, we can deal with the WMF-QKF. The detailed algorithm is omitted here.

Remark 7. For the case of multiple dimension measurements of each sensor, $H_{i}(t)$ is assumed to be full row rank. If not, the full-rank decomposition can be implemented. Then the measurement of each sensor can be compressed to a reduced dimension measurement without information loss. Or other compressed algorithms [21, 22] can be used for the multiple dimension measurements of each sensor. Then its each component is quantized and transmitted. Thus, the bandwidth can be saved.

\section{Simulation Research}

Consider a discrete-time system measured by five sensors:

$$
\begin{gathered}
x(t+1)=\left[\begin{array}{ccc}
0.9226 & -0.633 & 0 \\
1 & 0 & 0 \\
0 & 1 & 0
\end{array}\right] x(t)+\left[\begin{array}{c}
0.5 \\
0 \\
0.2
\end{array}\right] w(t) \\
y_{i}(t)=h_{i} x(t)+v_{i}(t), \quad i=1,2,3,
\end{gathered}
$$

where $y_{i}(t)$ is the measurement signal and $v_{i}(t)$ is the measurement noise with mean zero and variance $\sigma_{v_{i}}^{2}$ and is independent with Gaussian noise $w(t)$ with mean zero and variance $\sigma_{w}^{2}$. Our goal is to find the WMF-QKF dependent on values (WMF-QKFV) of $\gamma_{i}(t)$ and WMF-QKF dependent on probabilities (WMF-QKFP) of $\gamma_{i}(t)$. In the simulation, we set noise variances $\sigma_{w}^{2}=1, \sigma_{v_{1}}^{2}=1, \sigma_{v_{2}}^{2}=2, \sigma_{v_{3}}^{2}=2.5$, $\sigma_{v_{4}}^{2}=2.5, \sigma_{v_{5}}^{2}=3$, measurement matrices $h_{1}=h_{2}=h_{3}=$

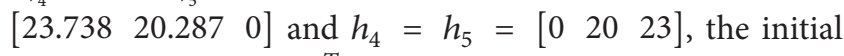
values $x(0)=\left[\begin{array}{lll}0 & 0 & 0\end{array}\right]^{T}$ and $P_{0}=0.1 I_{3}$, where $I_{3}$ is a $3 \times 3$ identity matrix, the bounds $\underline{U}_{1}=\underline{U}_{2}=\underline{U}_{3}=\underline{U}_{4}=\underline{U}_{5}=-40$ and $\bar{U}_{1}=\bar{U}_{2}=\bar{U}_{3}=\bar{U}_{4}=\bar{U}_{5}=40$ for measurements of five sensors, the initial bandwidths $b_{1}=b_{2}=b_{3}=b_{4}=b_{5}=1$, and the probabilities $\alpha_{1}=0.5, \alpha_{2}=0.7, \alpha_{3}=0.8, \alpha_{4}=0.8$ and $\alpha_{5}=0.7$ and we take 100 sampling data.

We solve the optimization problem (3) with the bits of the whole bandwidth $B=12$. We can compute the bandwidths $b_{1}=3, b_{2}=3, b_{3}=2, b_{4}=2$, and $b_{5}=2$. Tracking performance of WMF-QKFV and WMF-QKFP is shown in Figure 2 where bold curves denote the true value, dotted curves denote the estimates of WMF-QKFV, and dashed curves denote the estimates of WMF-QKFP. We see that WMF-QKFV has better accuracy than WMF-QKFP under the same bandwidth constraint. The comparison of mean square errors $\mathrm{MSE}_{k}=\left(\sum_{i=1}^{500}\left(\hat{x}_{k}^{(i)}(t \mid t)-x_{k}(t)\right)^{2}\right) / 500$ by 500 times Monte-Carlo test, $k=1,2,3$, denoting the $k$ th component of the state of all LFs, WMF-QKFV, and WMF-QKFP 

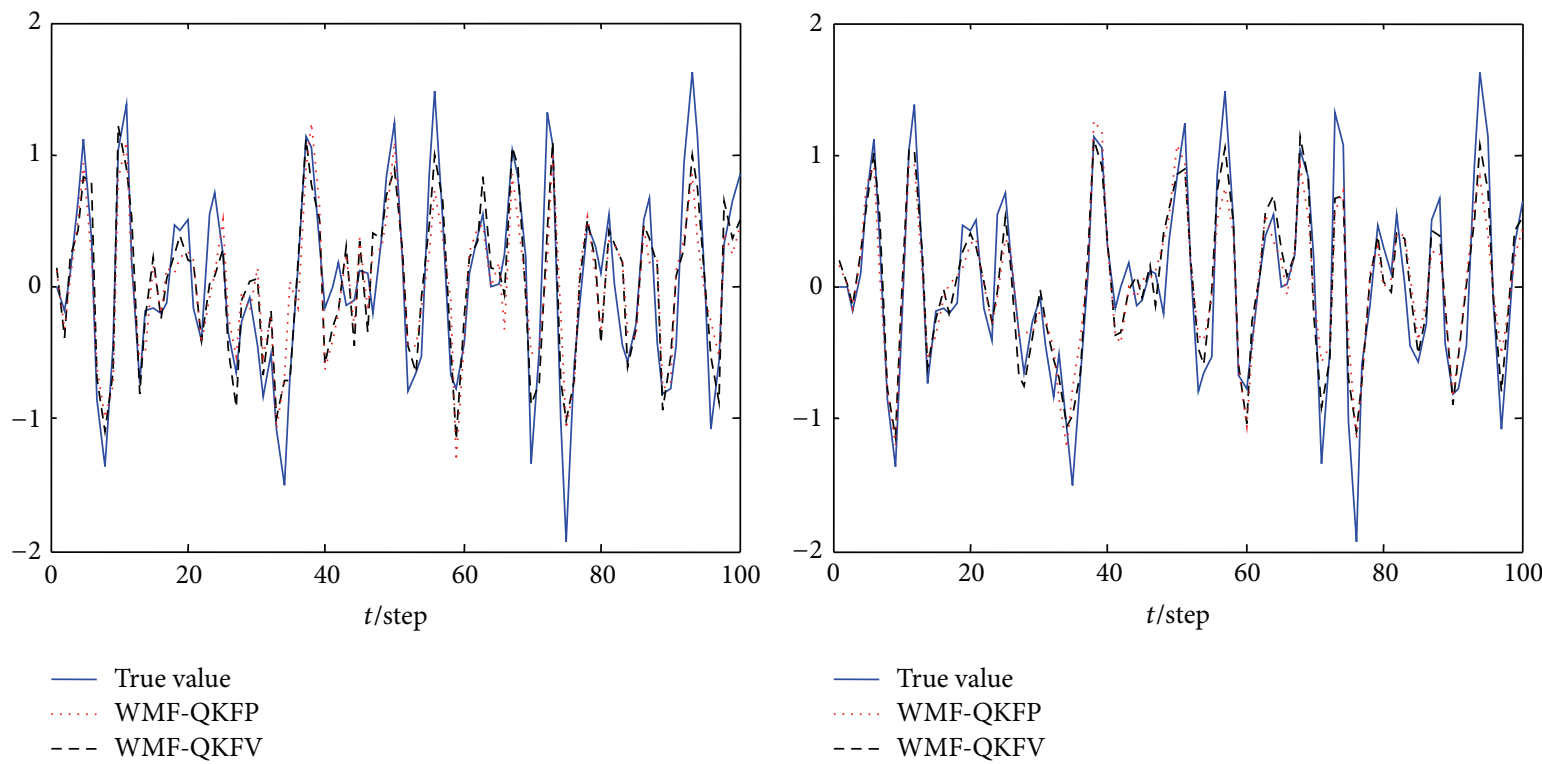

(a) The first state component

(b) The second state component

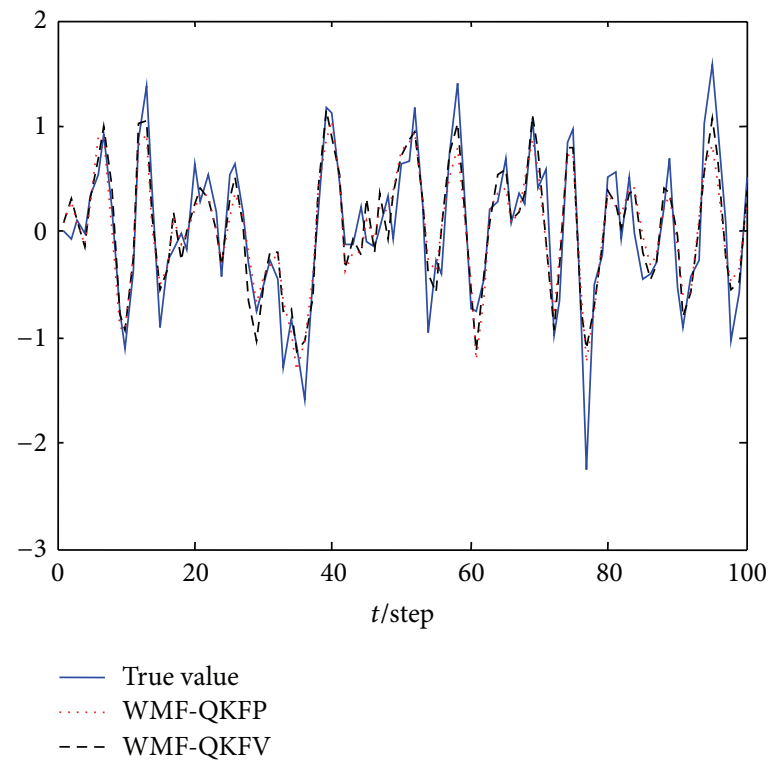

(c) The third state component

FIgURE 2: Comparison of tracking for WMF-QKFP and WMF-QKFV under whole bandwidth $B=12$.

is shown in Figure 3. We see that WMF-QKFP and WMFQKFV have better accuracy than any local filter and WMFQKFV has better accuracy than WMF-QKFP. All simulations verify the effectiveness of the proposed algorithms.

\section{Conclusion}

The weighted measurement fusion quantized filtering problem is investigated in a sensor network with bandwidth constraint and imperfect channels of missing measurements. Using the knowledge of whether a measurement is lost at the present time or the probabilities of missing measurements, two weighted measurement fusion quantized Kalman filters are developed based on the quantized measurements received, respectively. They have the same accuracy as the corresponding centralized fusion estimators and have the reduced computational cost.

\section{Conflict of Interests}

The authors declare that there is no conflict of interests regarding the publication of this paper.

\section{Acknowledgments}

This work is supported by the Natural Science Foundation of China (NSFC-61174139), by Chang Jiang Scholar 


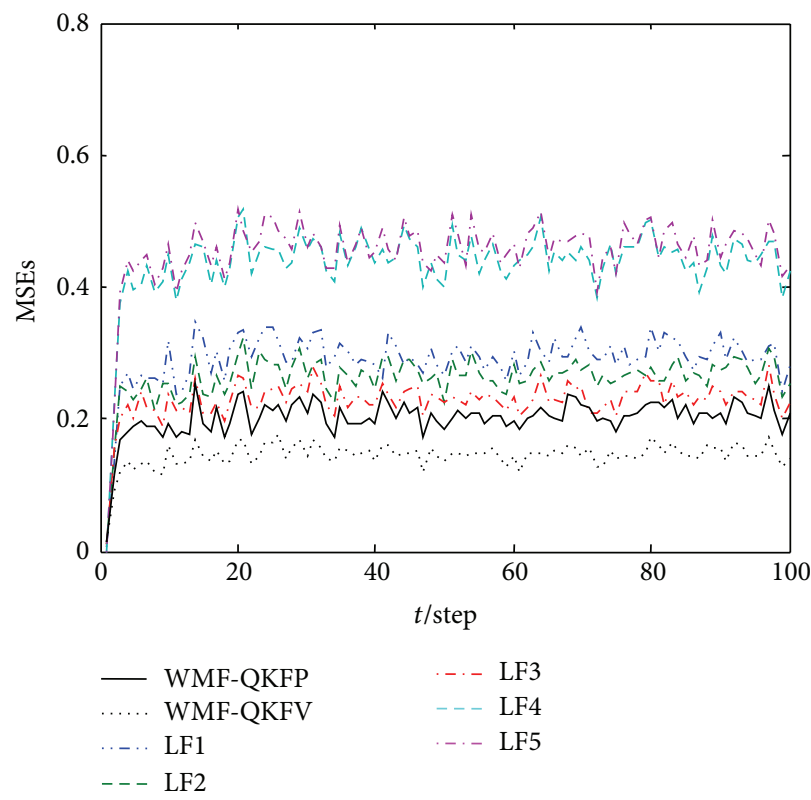

(a) The first state component

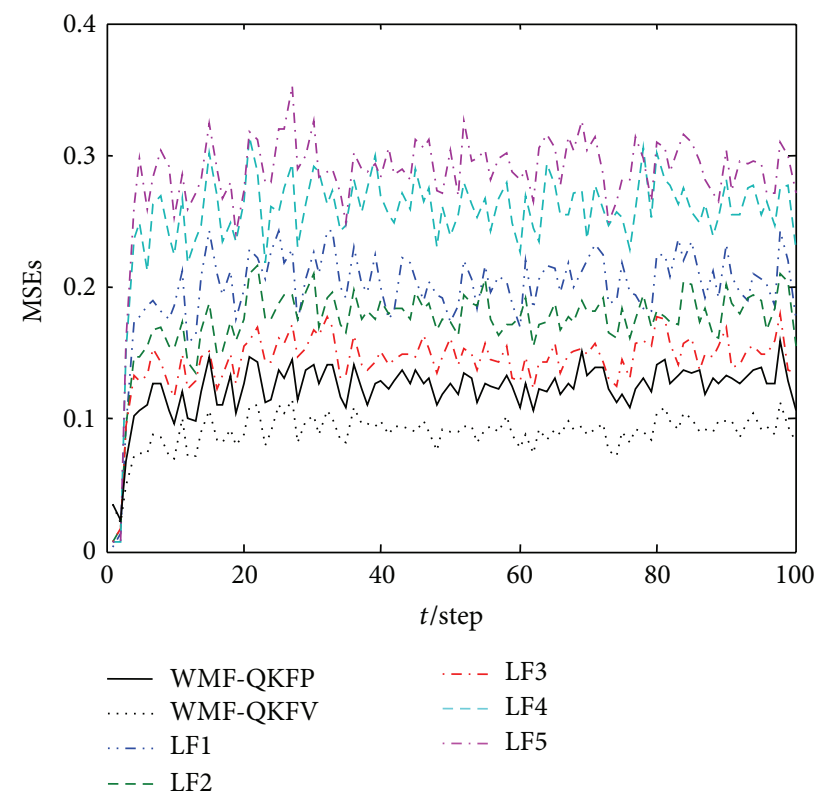

(b) The second state component



(c) The third state component

FIGURE 3: Comparison of accuracy of WMF-QKFP, WMF-QKFV, and all LFs under whole bandwidth $B=12$ by 100 times Monte-Carlo test.

Candidates Program for Provincial Universities in Heilongjiang (no. 2013CJHB005), by Science and Technology Innovative Research Team in Higher Educational Institutions of Heilongjiang Province (no. 2012TD007), by the Program for New Century Excellent Talents in University for Heilongjiang Province (no. 1154-NCET-01), by the Program for High-qualified Talents (no. Hdtd2010-03), and by Electronic Engineering Provincial Key Laboratory.

\section{References}

[1] C.-Y. Chong and S. P. Kumar, "Sensor networks: evolution, opportunities, and challenges," Proceedings of the IEEE, vol. 91, no. 8, pp. 1247-1256, 2003.
[2] E. F. Nakamura, A. A. F. Loureiro, and A. C. Frery, "Information fusion for wireless sensor networks: methods, models, and classifications," ACM Computing Surveys, vol. 39, no. 3, Article ID 1267073, 2007.

[3] X. Ning and C. G. Cassandras, "On maximum lifetime routing in wireless sensor networks," in Proceedings of the 48th IEEE Conference on Decision and Control, pp. 3757-3762, Shanghai, China, December 2009.

[4] M. Cardei and J. Wu, "Energy-efficient coverage problems in wireless ad-hoc sensor networks," Computer Communications, vol. 29, no. 4, pp. 413-420, 2006.

[5] J. J. Xiao and Z. Q. Luo, "Universal decentralized detection in a bandwidth-constrained sensor network," IEEE Transactions on Signal Processing, vol. 53, no. 8, pp. 2617-2624, 2005. 
[6] D. A. Castanon and D. Teneketzis, "Distributed estimation algorithms for nonlinear systems," IEEE Transactions on Automatic Control, vol. 30, no. 5, pp. 418-425, 1985.

[7] A. S. Willsky, M. G. Bello, D. A. Castanon, B. C. Levy, and G. C. Verghese, "Combining and updating of local estimates and regional maps along sets of one-dimensional tracks," IEEE Transactions on Automatic Control, vol. 27, no. 4, pp. 799-813, 1982.

[8] Z. Chair and P. K. Varshney, "Distributed Bayesian hypothesis testing with distributed data fusion," IEEE Transactions on Systems, Man and Cybernetics, vol. 18, no. 5, pp. 695-699, 1988.

[9] Z.-Q. Luo, "Universal decentralized estimation in a bandwidth constrained sensor network," IEEE Transactions on Information Theory, vol. 51, no. 6, pp. 2210-2219, 2005.

[10] Z.-Q. Luo, "An isotropic universal decentralized estimation scheme for a bandwidth constrained ad hoc sensor network," IEEE Journal on Selected Areas in Communications, vol. 23, no. 4, pp. 735-744, 2005.

[11] J.-J. Xiao, S. Cui, Z.-Q. Luo, and A. J. Goldsmith, "Power scheduling of universal decentralized estimation in sensor networks," IEEE Transactions on Signal Processing, vol. 54, no. 2, pp. 413-422, 2006.

[12] A. Ribeiro, G. B. Giannakis, and S. I. Roumeliotis, "SOI-KF: distributed Kalman filtering with low-cost communications using the sign of innovations," IEEE Transactions on Signal Processing, vol. 54, no. 12, pp. 4782-4795, 2006.

[13] K. You, L. Xie, S. Sun, and W. Xiao, "Multiple-level quantized innovation Kalman filter," in Proceedings of the 17th World Congress, International Federation of Automatic Control (IFAC '08), pp. 1420-1425, July 2008.

[14] K. You, L. Xie, S. Sun, and W. Xiao, "Quantized filtering of linear stochastic systems," Transactions of the Institute of Measurement and Control, vol. 33, no. 6, pp. 683-698, 2011.

[15] M. Fu and C. E. de Souza, "State estimation for linear discretetime systems using quantized measurements," Automatica, vol. 45, no. 12, pp. 2937-2945, 2009.

[16] M. Fu and L. Xie, "The sector bound approach to quantized feedback control," IEEE Transactions on Automatic Control, vol. 50, no. 11, pp. 1698-1711, 2005.

[17] S. Sun, J. Lin, L. Xie, and W. Xiao, "Quantized Kalman filtering," in Proceedings of the IEEE 22nd International Symposium on Intelligent Control (ISIC '07), pp. 7-12, October 2007.

[18] N. Liu, J. Ma, and S. L. Sun, "Quantized Kalman filter for sensor networks with random packet dropouts," Advanced Materials Research, vol. 219-220, pp. 1040-1044, 2011.

[19] B. D. O. Anderson and J. B. Moore, Optimal Filtering, PrenticeHall, Englewood Cliffs, NJ, USA, 1979.

[20] X. Wang, S. Sun, K. Ding, and J. Xue, "Weighted measurement fusion white noise deconvolution filter with correlated noise for multisensor stochastic systems," Mathematical Problems in Engineering, vol. 2012, Article ID 257619, 16 pages, 2012.

[21] K. Zhang, X. R. Li, P. Zhang, and H. Li, "Optimal linear estimation fusion-part 6: sensor data compression," in Proceedings of the International Conference on Information Fusion, pp. 221-228, 2003.

[22] E. Song, Y. Zhu, and J. Zhou, "Sensors' optimal dimensionality compression matrix in estimation fusion," Automatica, vol. 41, no. 12, pp. 2131-2139, 2005. 


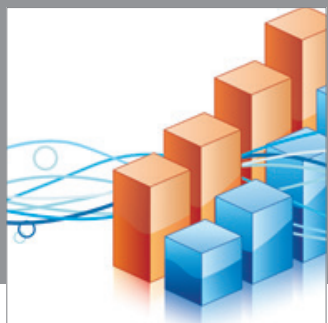

Advances in

Operations Research

mansans

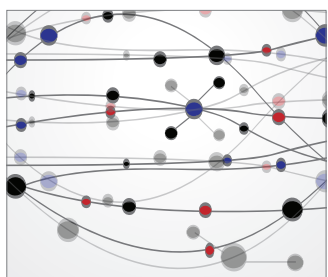

The Scientific World Journal
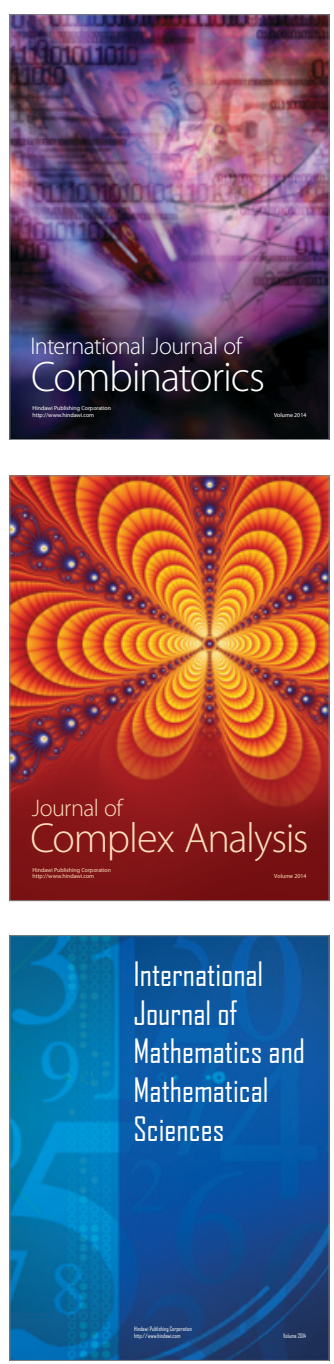
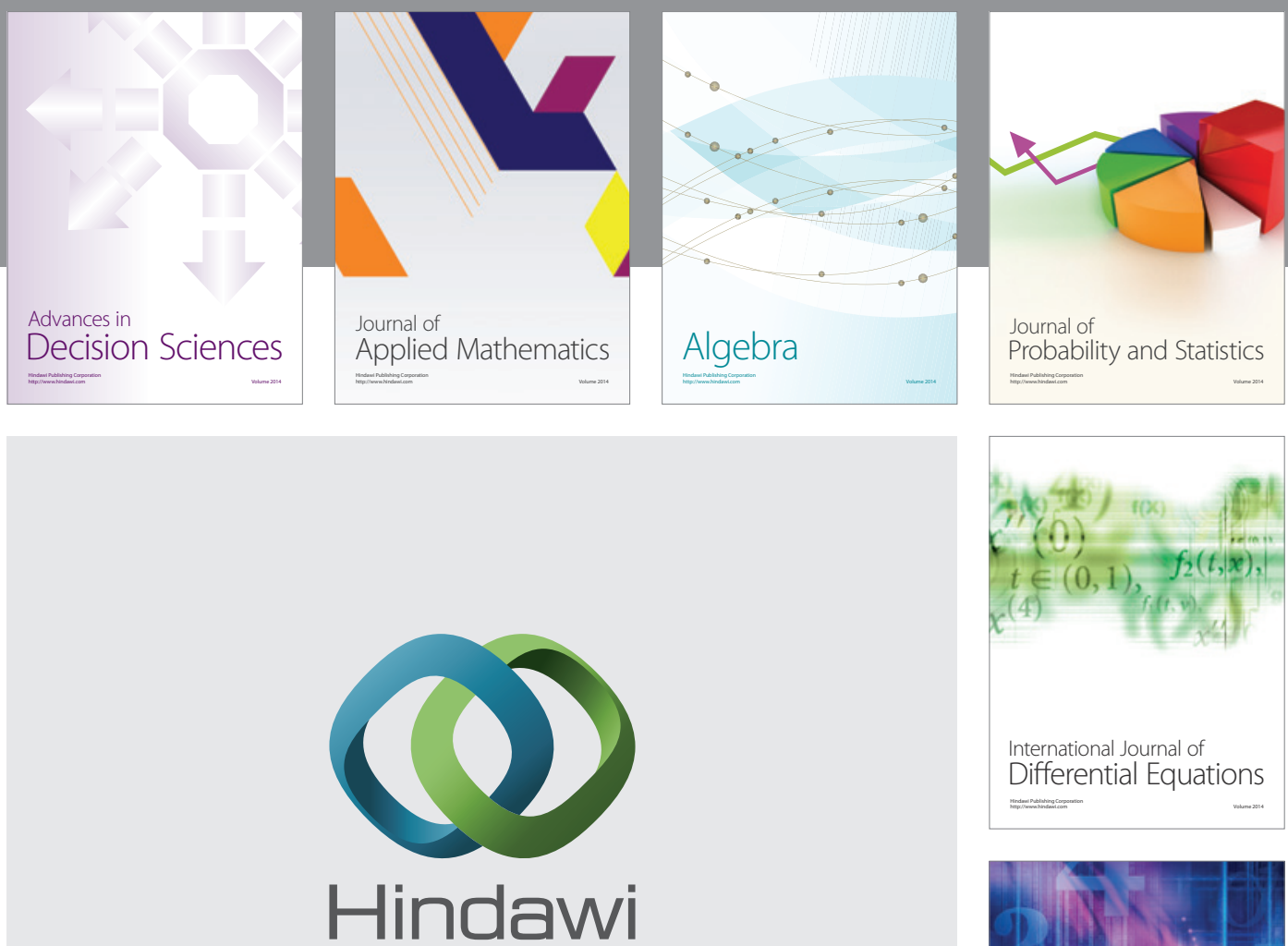

Submit your manuscripts at http://www.hindawi.com
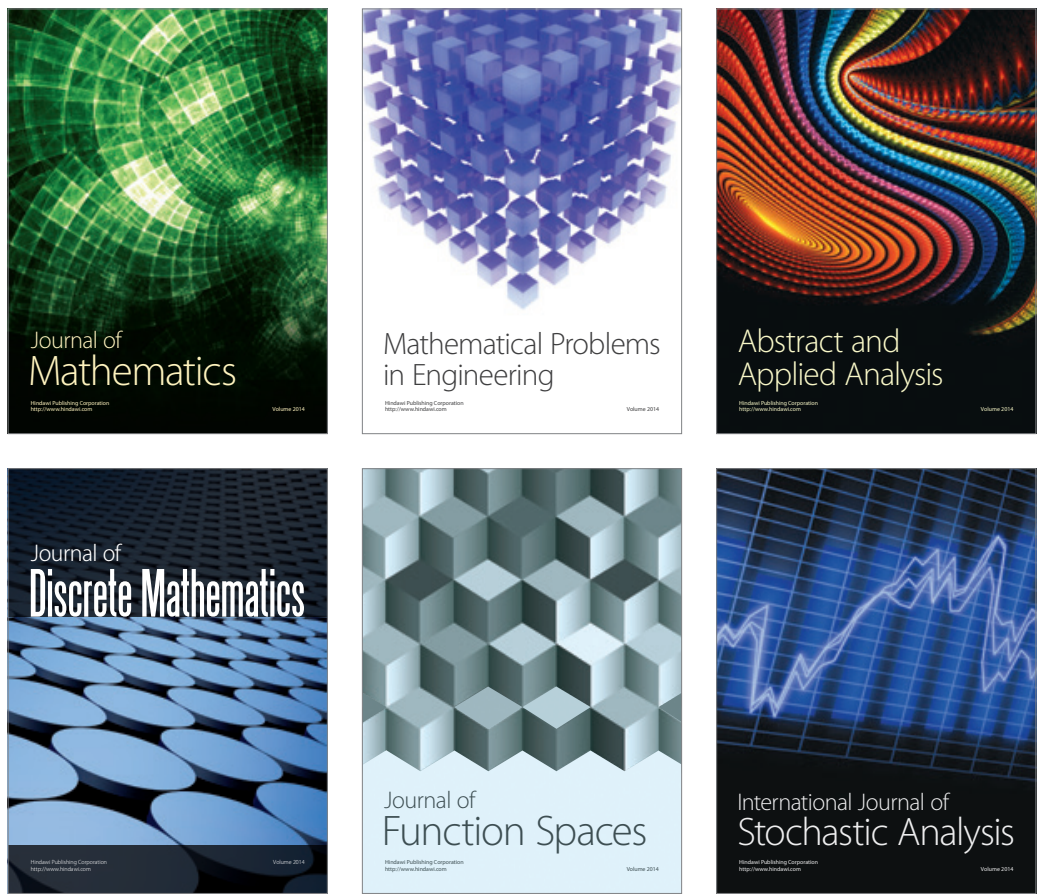

Journal of

Function Spaces

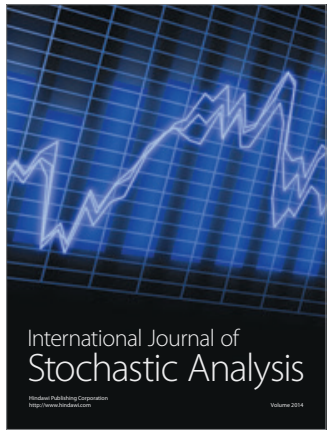

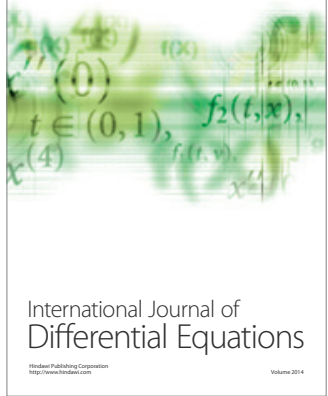
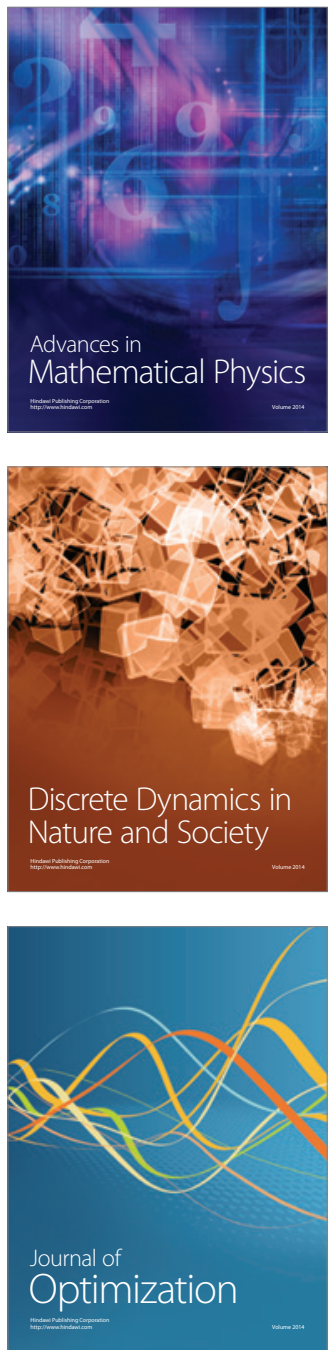